\title{
Evaluation of the diagnostic accuracy of transvaginal sonography versus hysteroscopy in detection of submucous myomas in peri and postmenopausal women with abnormal uterine bleeding
}

\author{
Kushla Pathania, Surbhi Sharma*
}

Department of Obstetrics and Gynecology, Indira Gandhi Medical College and Hospital, Shimla, Himachal Pradesh, India

Received: 10 August 2021

Revised: 13 September 2021

Accepted: 14 September 2021

\section{*Correspondence:}

Dr. Surbhi Sharma,

E-mail: surbhisharma2425@gmail.com

Copyright: () the author(s), publisher and licensee Medip Academy. This is an open-access article distributed under the terms of the Creative Commons Attribution Non-Commercial License, which permits unrestricted non-commercial use, distribution, and reproduction in any medium, provided the original work is properly cited.

\section{ABSTRACT}

Background: Abnormal uterine bleeding is a very common gynaecological condition that affects all age groups. This study was aimed at assessing the usefulness of TVS in comparison with hysteroscopy in AUB evaluation. Aim was to evaluate the diagnostic accuracy of transvaginal sonography versus hysteroscopy in detection of submucous myomas in peri and postmenopausal women with abnormal uterine bleeding.

Methods: The present study was prospective cross sectional study conducted in the Department of Obstetrics and Gynaecology, Kamla Nehru State Hospital for Mother and Child Indira Gandhi Medical College Shimla for period of one year w.e.f. $1^{\text {st }}$ May 2018 to $30^{\text {th }}$ April 2019. The study was started after hospital ethical committee approval. 76 patients peri and post-menopausal women were enrolled in the study after taking written consent.

Results: On TVS- all the study subjects underwent TVS examination, submucous fibroid was detected in 10 subjects $(n=76)$ i.e. $13.1 \%$ (all perimenopausal). On hysteroscopy submucous fibroid was detected in $11(14.4 \%)$ subjects, on histopathology it was confirmed in 11 subjects (14.4\%). Out of 11, 8 patients underwent hysterectomy, 1 myomectomy, 2 patients had hysteroscopic removal of fibroid. 1 subject with AUB-LSM was wrongly diagnosed as AUB-P. Sensitivity, specificity, positive and negative predictive values of transvaginal sonography versus hysteroscopy was $90.09 \%, 100 \%, 100 \%, 98.5 \%$ respectively.

Conclusions: TVS is an important initial modality for evaluating the patient of AUB. It is quick, simple, painless, least invasive, less expensive and readily available procedure and does not need full bladder like TAS. On the other hand, hysteroscopy has a better diagnostic accuracy as it provides the option of see and treat which is recommended for peri and post-menopausal women with AUB.

Keywords: Abnormal uterine bleeding, Hysteroscopy, Submucous fibroid, Transvaginal sonography

\section{INTRODUCTION}

Abnormal uterine bleeding is a very common gynaecological condition that affects all age groups. One third of patients attending gynaecology OPD present with complaints of abnormal uterine bleeding. ${ }^{1}$ Bleeding is said to be abnormal when the pattern is irregular, of abnormal duration (>7 days), or abnormal amount $(>80 \mathrm{ml}){ }^{2}$ Accurate diagnosis of the cause of AUB can reduce the frequency of hysterectomy. This study was aimed at assessing the usefulness of TVS in comparison with hysteroscopy in AUB evaluation. ${ }^{3}$

During climacteric, ovarian activity declines. Initially, ovulation fails, no corpus luteum forms, and no progesterone is secreted by the ovary. Therefore, the premenopausal menstrual cycles are shortened, often anovulatory and irregular. The irregularity in menstrual 
cycle during perimenopause can be due to anovulation or to irregular maturation of follicles. ${ }^{4}$ The increased risk of endometrial hyperplasia and endometrial carcinoma is more evident in peri-menopausal and post-menopausal women with abnormal uterine bleeding. ${ }^{5}$

During the last decades, several methods including transvaginal ultrasonography, saline infusion sonography, and hysteroscopy, have been developed to assess uterine cavity, with their own advantages and disadvantages. Although TVS is a simple examination allowing clear visualization of most uterine conditions, several concerns have been raised regarding its accuracy. ${ }^{6}$ Hysteroscopy on the other hand, allows direct visualization and sampling of the uterine cavity and has an established diagnostic value for many uterine conditions. However, the latter modality is not as cost-effective and convenient as ultrasonographic imaging modalities, which are associated with relatively less patient discomfort and do not necessitate anesthesia. Invasive nature of Hysteroscopy and high cost preclude its use as an initial diagnostic procedure in patients with AUB. ${ }^{7}$

Recently transvaginal sonography (TVS) has permitted the use of higher frequency ultrasound waves at greater proximity to the uterus. It is relatively cheap, needs no anaesthesia and being non-invasive, can be a first diagnostic step in evaluation of abnormal uterine bleeding. ${ }^{8,9}$

Diagnostic hysteroscopy has ushered a new era in the evaluation of abnormal uterine bleeding and is accepted as the gold standard for determining the cause of endometrial pathologies presenting with the same. ${ }^{8,9}$ Hysteroscopic examination is superior to other methods in evaluation of endometrial cavity. It should not replace the histological diagnosis. Hysteroscopy is complementary to histological analysis as it permits pan evaluation of endometrial mucosa and biopsy is taken from abnormal areas in the same setting. ${ }^{10}$

\section{METHODS}

The present study was prospective cross sectional study conducted in the Department of Obstetrics and Gynaecology, Kamla Nehru State Hospital for Mother and Child Indira Gandhi Medical College Shimla for period of one year w.e.f. $1^{\text {st }}$ May 2018 to $30^{\text {th }}$ April 2019. The study was started after hospital ethical committee approval.

\section{Inclusion criteria}

Perimenopausal women age $>40$ years with menorrhagia, menometrorhagia, polymenorrhea, intermenstrual bleeding, post coital bleeding, post-menopausal bleeding.

\section{Exclusion criteria}

Pregnancy, woman taking hormonal replacement therapy, obvious cause of bleeding from cervix and vagina, woman with bleeding diathesis, woman on anticoagulant therapy, transvaginal sonography showing adenexal pathology.

76 patients ( $\geq 40$ years and post-menopausal women were enrolled in the study (after taking written consent). A detailed history, general and systemic examination was done. The study population was subjected to a thorough physical examination and routine investigations (haemoglobin, $\mathrm{ABO}$ and $\mathrm{Rh}$, blood sugar, urine routine and microscopy) followed by transvaginal sonography and then by hysteroscopy after obtaining post-counselling informed consent. The procedure was performed in a minor operation theatre under sedation. Patient was taken to the procedure room and placed in the dorsal lithotomy position. After a bimanual examination, a bivalved or weighted speculum was used to bring the cervix into view. The cervix was cleaned with using of $10 \%$ povidoneiodine or a single-toothed tenaculum was then applied to the anterior lip of the cervix. A small amount of local anaesthetic was used prior to applying the tenaculum.

Normal saline was used as the distension media. Once equipment for distention media was activated and functional, the flow of the medium was started. As the hysteroscope was introduced to the external cervical os and advanced into the endocervical canal, attention was turned to the video monitor or eyepiece. The distal tip of the hysteroscope was then gently advanced through the length of the cervix, taking care to keep the endocervical canal central on the viewing field when using a $0_{-}$scope. The first evaluation was done in panoramic view of the intrauterine cavity. Next, careful inspection of the following areas was done: lateral uterine walls, superior uterine cavity, and anterior and posterior uterine walls. Gentle movement of the hysteroscope was done during the procedure. Excessive trauma to the endometrial surface was avoided as it may cause bleeding which might obscure the view and has risk of perforation. Any pathology was inspected and documented.

Guided biopsy was taken and subjected to histopathological evaluation. Chi square test was applied to test the strength of association and $p$ value less than 0.05 was considered significant.

For quantitative data, mean and standard deviation was calculated and comparison will be done using student ttest.

\section{RESULTS}

The present study aimed to evaluate the diagnostic accuracy of TVS versus hysteroscopy in detection of submucous myomas in peri and postmenopausal women and correlating the diagnosis with histopathology. A total of 76 symptomatic perimenopausal and post-menopausal women were included in the study at Department of Obstetrics and Gynaecology, Kamla Nehru State Hospital for Mother and Child, Indira Gandhi Medical College Shimla over a period of one year. 
All the study subjects underwent TVS examination. Submucous fibroid was detected in 10 subjects $(n=76)$ i.e. $13.1 \%$ (all perimenopausal).

Table 1: TVS findings.

\begin{tabular}{|lll|}
\hline TVS findings & N & Percentage \\
\hline Submucous fibroid & 10 & 13.1 \\
\hline
\end{tabular}

\section{Hysteroscopy findings}

In the present study, submucous fibroid was detected in 11 $(14.4 \%)$ subjects. Hysteroscopy findings $(n=76)$.

Table 2: Hysteroscopy findings.

\begin{tabular}{|lll|}
\hline Hysteroscopy findings & N & Percentage \\
\hline Submucous fibroid & 11 & 14.4 \\
\hline
\end{tabular}

\section{Histopathology examination}

On histopathology, submucous fibroid was detected in 11 (14.4\%) subjects.

Table 3: Histopathology examination.

\begin{tabular}{|lll|}
\hline Hystopathology findings & N & Percentage \\
\hline Submucous fibroid & 11 & 14.4 \\
\hline
\end{tabular}

Table 4: Correlation of TVS, hysteroscopy with histopathology submucous fibroid.

\begin{tabular}{|llll|}
\hline & Peri & Post & Total \\
\hline TVS & 10 & nil & $13.1 \%$ \\
\hline Hysteroscopy & 10 & 1 & $14.4 \%$ \\
\hline HPE & 10 & 1 & $14.4 \%$ \\
\hline
\end{tabular}

TVS diagnosed 10 subjects $(13.1 \%)$ (all perimenopausal) as fibroid whereas hysteroscopy revealed 11 cases $(14.4 \%)$ of fibroid. After final diagnosis i.e. histopathology out of 11, 8 patients underwent hysterectomy, 1 myomectomy, 2 patients had hysteroscopic removal of fibroid.

1 subject with AUB-LSM was wrongly diagnosed as AUB-P.

Table 5: Diagnostic value.

\begin{tabular}{|lllll|}
\hline $\begin{array}{l}\text { TVS versus } \\
\text { hysteroscopy }\end{array}$ & Sensitivity & Specificity & PPV & NPV \\
\hline $\begin{array}{l}\text { Submucous } \\
\text { fibroid }\end{array}$ & $90.9 \%$ & $100 \%$ & $100 \%$ & $98.5 \%$ \\
\hline
\end{tabular}

\section{DISCUSSION}

Abnormal uterine bleeding is defined as bleeding from uterus that differs from that of usual normal menstrual bleeding, in frequency of occurrence or in amount or in alteration of flow. In the past, when few diagnostic options were available, this condition was routinely managed with uterine curettage. $\mathrm{D}$ and $\mathrm{C}$ being a blind procedure can miss the specific lesions. However, with the addition of transvaginal sonography, saline infusion sonography and hysteroscopy diagnostic accuracy has improved significantly. Office hysteroscopy has been shown to be a simple, safe, well tolerated and reliable procedure in the diagnosis of AUB across all age groups and its wide spread use can drastically reduce the need for conventional curettage. In the present study the diagnostic accuracy of TVS and hysteroscopy was compared and correlated with histopathological findings in women with peri and postmenopausal AUB.

Table 6: TVS versus HPE submucous fibroid.

\begin{tabular}{|llll|}
\hline Study & Year & Sensitivity & Specificity \\
\hline Veena et al & 2005 & $75 \%$ & $98 \%$ \\
\hline Shukla et al & 2012 & $55 \%$ & $96.08 \%$ \\
\hline Present study & 2018 & $90 \%$ & $100 \%$ \\
\hline
\end{tabular}

Specificity of TVS for diagnosing uterine submucous fibroid was $100 \%$ in the present study. Similarly Veena et al and Shukla et al have documented 98\% and 96.08\% specificity of TVS respectively for fibroid when correlated with histopathological findings. Sensitivity of TVS for uterine fibroid was $90 \%$ in the present study as compared to sensitivity of $55 \%$ observed in a study by Shukla et al and $75 \%$ sensitivity observed by Veena et al. The difference could be due to difference in the percentage of patients in AUB-L i.e. 13.1\% in the present study, 6.6\% in the study by Veena et al and $11.7 \%$ in the study by Shukla et al.

Table 7: Submucous fibroid hysteroscopy versus HPE.

\begin{tabular}{|llll|}
\hline Study & Year & Sensitivity & Specificity \\
\hline Shukla et al & 2012 & $100 \%$ & $100 \%$ \\
\hline Present study & 2018 & $100 \%$ & $100 \%$ \\
\hline
\end{tabular}

In a study by Shukla et al it was observed that sensitivity of hysteroscopy for fibroid was $100 \%$ which is similar to the present study. The present study observed specificity of $100 \%$ which is comparable to study by Shukla et al i.e. $100 \%$. In the present study on hysteroscopy 11 cases were diagnosed as submucous fibroid out of these eleven cases eight underwent hysterectomy, one myomectomy and two patients had hysteroscopic removal of fibroid. The final sensitivity and specificity of hysteroscopy for submucous fibroid was $100 \%$.

Thus, hysteroscopy is complementary to histological analysis.

\section{CONCLUSION}

Abnormal uterine bleeding is a problem which women in perimenopausal and postmenopausal period frequently encounter. TVS is an important modality for evaluating the 
patient of AUB. It is quick, simple, painless, least invasive, less expensive and readily available procedure and does not need full bladder like TAS. TVS can be the most cost effective initial test in women with abnormal uterine bleeding especially in perimenopausal age group but its ability for screening the lesions in the endometrial cavity is limited. Hysteroscopy has a better diagnostic accuracy as it provides the option of see and treat which is recommended for peri and post-menopausal women with AUB. Also hysteroscopy can detect intracavitary lesions such as leiomyomas and polyps that might be missed on TVS or endometrial sampling. Hence it should be made an essential part of diagnostic workup of abnormal uterine bleeding.

Funding: No funding sources Conflict of interest: None declared

Ethical approval: The study was approved by the Institutional Ethics Committee

\section{REFERENCES}

1. Awwad JT, Toth TL, Schiff I. Abnormal uterine bleeding in the perimenopause. Int J Fertil Menopaus Stud. 1993;38(5):261-9.

2. Speroff L, Fritz MA. Menopause and the perimenopausal transition. In: Clinical gynaecologic endocrinology and infertility. 7th edn. Philadelphia: Lippincott Williams and Wilkins; 2005:621-688.

3. Goyal BK, Gaur I, Sharma S, Saha A, Das NK. Transvaginal sonography versus hysteroscopy in evaluation of abnormal uterine bleeding. Med J Armed Forces India. 2015;71(2):120-5.

4. Padubidri VG, Daftary S. Perimenopause, Menopause, Premature Menopause and
Postmenopausal bleeding. In: Shaw's Textbook of Gynaecology. 16th edn. New Delhi: Elsevier; 2015:65-78.

5. Kumar A, Mittal S. Endometrial sampling: how and why. Obstet Gynaecol Today. 2007;12(6):284-7.

6. Vitner D, Filmer S, Goldstein I, Khatib N, Weiner Z. A comparison between ultrasonography and hysteroscopy in the diagnosis of uterine pathology. Eur J Obstet Gynaecol Reprod Biol. 2013;171(1):1435.

7. Dreisler E, Poulsen LG, Antonsen SL, Ceausu I, Depypere H, Erel CT, et al. EMAS clinical guide: assessment of the endometrium in peri and postmenopausal women. Maturitas. 2013;75:181-90.

8. Dijkhuizen FP, Brolmann HA, Potters AE, Bongers MY, Heinz AP. The accuracy of transvaginal ultrasonography in the diagnosis of endometrial abnormalities. Obstet Gynecol. 1996;87:345-9.

9. Emanuel MH, Warmstaker $\mathrm{K}$, Lannes FB. A prospective comparison of transvaginal sonography and diagnostic hysteroscopy in evaluation of patients with abnormal uterine bleeding. Am J Obstet Gynecol. 1995;172:547-52.

10. Barman SC, Bardhan J, Roy S, Sark NK. Comparative evaluation of transvaginal sonography and diagnostic hysteroscopy in abnormal uterine bleeding in perimenopausal age with their histopathological correlation. Sch J App Med Sci. 2017;5(3B):838-43.

Cite this article as: Pathania K, Sharma S.

Evaluation of the diagnostic accuracy of transvaginal sonography versus hysteroscopy in detection of submucous myomas in peri and postmenopausal women with abnormal uterine bleeding. Int J Reprod Contracept Obstet Gynecol 2021;10:3885-8. 